\title{
The Mid-Infrared Properties of Embedded Super Star Clusters in He2-10 and IIZw40
}

\author{
I.Sakon ${ }^{1}$, T. Onaka ${ }^{1}$, H. Takahashi ${ }^{2}$, T. Miyata ${ }^{1}$, S. Sako ${ }^{1}$, Y. Y.

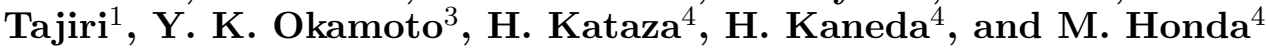

${ }^{1}$ University of Tokyo, Japan, ${ }^{2}$ Gunma Astronomical Observatory, Japan, ${ }^{3}$ Ibaraki University,

${ }^{4}$ Institute of Space and Astronautical Science, Japan Aerospace Exploration Agency, Japan

We observed embedded super-star clusters (SSCs) in blue compact dwarf galaxies (BCDs) IIZw40 and He2-10 with N-band and Q-band imaging and spectroscopy using the Cooled Mid-Infrared Camera and Spectrometer (COMICS) on board the $8 \mathrm{~m}$ Subaru Telescope. Our infrared images of Henize 2-10 resolve the radio knots (knots I-V; Kobulnicky \& Johnson 1999) and the N- and Q-band spectra show the strong [NeII] $12.8 \mu \mathrm{m}$ and the [SIII] $18.71 \mu \mathrm{m}$ together with relatively weak [SIV] $10.51 \mu \mathrm{m}$ and [ArIII] $8.99 \mu \mathrm{m}$ as well as the distinct unidentified infrared (UIR) bands at 8.6, 11.2 and $12.7 \mu \mathrm{m}$. We find the typical stellar spectral type of the members in the SSCs is O9 using the CoStar model (Stasinska \& Schaerer 1997) and that the ionization environment in the most deeply embedded SSC(POS\#D) is the softest among those SSCs. The UIR bands exhibit a flat distribution over the galaxy and do not show a correlation with the MIR continuum, suggesting that polycyclic aromatic hydrocarbons (PAHs) are not strongly associated with the SSCs. We also find a possible decrease in the UIR $12.7 \mu \mathrm{m} / 11.2 \mu \mathrm{m}$ ratio inside the embedded SSCs, suggesting that smooth-edged large PAHs are dominant (Hony et al. 2001) there. The small UIR to continuum ratio inside the embedded SSCs also suggests that the size distribution of the carbonaceous dust is skewed to the larger ones inside the embedded SSCs. Possible destruction of smaller species by harsh radiation field in the vicinity of massive stars due to the smaller heat capacity and/or the coagulation process that have been undertaken in dense dusty embedded SSCs would explain our results consistently. From the mid-infrared spectroscopy of IIZw40, the UIR bands are detected only in the south-western border of the mid-infrared peaks, suggesting the uneven distribution of PAHs within $4 \times 10^{6} \mathrm{yr}$ (Vanzi et al. 1996) after the nuclear starburst triggered in the merging of two small original galaxies.
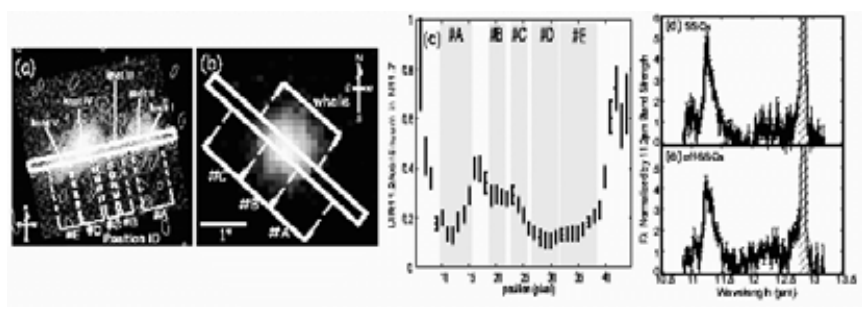

Figure 1. (a),(b) $11.7 \mu \mathrm{m}$ image of He 2-10 and IIZw40 and the slit position for N-band low-res spectroscopy. (c) Spatial variations in the ratio of the UIR $11.2 \mu \mathrm{m}$ feature to the continuum emission in the N11.7 band in He $2-10$. (d), (e) averaged UIR spectra of OOP bending modes inside and outside the embedded SSCs.

This work is based on data collected at Subaru Telescope operated by the National Astronomical Observatory in Japan. I.S. is financially supported by the Japan Society for Promotion of Science (JSPS).

\section{References}

Hony, S. et al. 2001, A\&A 370, 1030

Kobulnicky, H. A., \& Johnson, K. W. 1999, ApJ 527, 154

Stasińska, G., \& Schaerer, D. 1997, A\&A 322, 615

Vanzi, L., Rieke, G. H., Martin, C. L., Shields, J. C. 1996, ApJ 466, 150 